\title{
Extent of cortical involvement in amyotrophic lateral sclerosis - an analysis based on cortical thickness
}

\author{
Johannes Thorns' ${ }^{1}$ Henk Jansma ${ }^{1}$, Thomas Peschel ${ }^{2}$, Julian Grosskreutz ${ }^{3}$, Bahram Mohammadi ${ }^{1}$, \\ Reinhard Dengler ${ }^{4}$ and Thomas F Münte ${ }^{1^{*}}$
}

\begin{abstract}
Background: Besides the defining involvement of upper and lower motor neurons, the involvement of extramotor structures has been increasingly acknowledged in amyotrophic lateral sclerosis (ALS).

Methods: Here we investigated a group of 14 mildly to moderately affected ALS patients and 14 age-matched healthy control participants using cortical thickness analysis. Cortical thickness was determined from high resolution 3D T1 magnetic resonance images and involved semiautomatic segmentation in grey and white matter, cortical alignment and determination of thickness using the Laplace method. In addition to a whole-cortex analysis a region of interest approach was applied.
\end{abstract}

Results: ALS patients showed regions of significant cortical thinning in the pre- and postcentral gyri bilaterally. Further regions of cortical thinning included superior and inferior parietal lobule, angular and supramarginal gyrus, insula, superior frontal, temporal and occipital regions, thus further substantiating extramotor involvement in ALS. A relationship between cortical thickness of the right superior frontal cortex and clinical severity (assessed by the ALS functional rating scale) was also demonstrated.

Conclusions: Cortical thickness is reduced in ALS not only in motor areas but in widespread non-motor cortical areas. Cortical thickness is related to clinical severity.

Keywords: ALS, Cortical thickness, MRI

\section{Background}

Amyotrophic lateral sclerosis (ALS) is a neurodegenerative disease with upper and lower motor neuron involvement as its clinical hallmarks. Dating back to very early observations that a significant percentage of ALS patients develop a dementia it has been clear that extramotor involvement can occur. That such involvement is the rule rather than the exception has been underscored by findings from neuropsychological [1-4] and neuroimaging [5-9] investigations. The recent discovery that the C9ORF72 hexanucleotide repeat can cause both, frontotemporal dementia and ALS as well as overlapping syndromes, further underscores that the molecular underpinnings of ALS

\footnotetext{
* Correspondence: Thomas.muente@neuro.uni-luebeck.de

'Department of Neurology, University of Lübeck, Ratzeburger Allee 160, 23538 Lübeck, Germany

Full list of author information is available at the end of the article
}

might damage extramotor and motor parts of the nervous system [10]. The question therefore arises, to what extent disturbances of non-motor functions are reflected in changes of cortical structure. Primary motor and extramotor involvement has been demonstrated repeatedly by voxel based morphometry (VBM) [11-13], although other studies did not report atrophy $[14,15]$.

VBM is a fully automated, operator-independent technique that allows the voxel-wise comparison of segmented grey and white matter images between two groups of subjects [16-19]. As such, it has the advantages of being fast and independent of any observer rating or a priori hypothesis on the location of the structural abnormality. There are several limitations of this approach, however $[18,20]$. For example, VBM simultaneously reflects size, position and morphology. As nonlinear spatial normalization and spatial filtering is used to accommodate anatomical 
variations between brains, there is the danger of missing important differences, in particular when these are circumscribed. Moreover, VBM reveals differences in grey matter density, which may not equate in a simple way to atrophy.

In the present investigation we therefore employed an alternative to VBM, cortical thickness analysis (CTA). CTA is based on a semiautomatic/interactive segmentation of brain white and grey matter followed by a determination of the thickness of the cortical layer [21-24]. Thickness values are then entered into standard between group statistics allowing a comparison for the entire cortical surface. Neuropathological studies have described atrophy involving the precentral gyrus with loss of Betz cells and gliosis in ALS $[25,26]$ which lead us to suspect a decreased cortical thickness in ALS. Also, cortical thinning of the motor cortex has been described recently in ALS [27-30] and primary lateral sclerosis [31]. Verstraete et al. $[28,29]$ could demonstrate that cortical thinning in temporal regions was related to faster clinical progression, whereas in a recent study by Agosta et al. [30] age and cortical thinning in sensorimotor cortex was related in ALS patients but not in controls. Interestingly, no relationship was found between clinical severity and cortical thickness. Finally, in a recent study by Schuster et al. [32] clinical variables were related to cortical thinning of the primary motor cortex (PMC). Upper motor neuron (UMN) signs in the bulbar region were associated with bilateral thinning within the bulbar segment on the motor cortex, whereas UMN signs of the extremities were associated with thinning in the limb segment of the motor cortex.

In the present investigation, we asked specifically whether other areas in addition to the motor cortex are affected with regard to cortical thinning. We were further interested in whether CTA is more sensitive to cortical changes in ALS than VBM and performed CTA on a sample of ALS patients and controls that has previously been reported in a VBM study [13].

\section{Methods}

\section{Patients}

The study was approved by the ethics committee of Hannover Medical School. All participants gave their written informed consent prior to their inclusion in the study.

The original patient group comprised 17 subjects (3 women) classified as having definite, probable or possible ALS according to the revised El Escorial criteria [33] at the time of testing. All but one patient had progressed to definite or probable ALS on follow up. Data quality of the scans did not allow reliable differentiation between grey and white matter in three patients. For the remaining 14 patients mean ( \pm S.D.) duration since onset of symptoms, henceforth disease duration, was
$23.1 \pm 7.7$ months, mean age was $58.4 \pm 13.1$ (range $31-77$ ) years and duration of education in years was 12.8 \pm 2.6 . Seventeen age matched healthy participants were recruited as a control group. Of these, only those 14 participants that most closely matched the 14 remaining patients with regard to age $(57.9 \pm 11.8, \mathrm{p}=0.86)$, sex (patients: 2 women, controls: 3 women), and education (controls $11.9 \pm 3.1, \mathrm{p}=0.79$ ) were further analyzed. Neither patients nor controls had a history of cerebrovascular disease, longstanding hypertension or inflammatory disease of the central nervous system. All patients received riluzole treatment but no psychoactive drugs. Their score on the revised ALS functional rating scale [34,35] (ALSFRS-R) was $40.6 \pm 4.7$ points.

\section{Clinical data}

A number of clinical data were obtained to correlate these with cortical thickness. ALSFRS-R progression rate per month was calculated for the total disease duration [PR/ $\mathrm{mth}(\mathrm{t})]$ and the six month period prior to MRI $[\mathrm{PR} / \mathrm{mth}$ (6)]. Sum scores of Medical Research Council (MRC) muscle strength were taken for the upper extremity (shoulder abduction, inward and outward rotation; elbow flexion and extension; lower arm pronation and supination; wrist flexion and extension; finger flexion, extension, abduction and adduction; 5th finger abduction and thumb opposition; highest possible score 75 points) and the lower extremity (hip strength; knee flexion and extension; foot flexion, extension, inversion and eversion; toe flexion and extension; best possible score 45 points). Bulbar involvement was described as a sum score of dysarthria $(0=$ no, $1=$ yes $)$, dysphagia $(0=$ no, $1=$ yes $)$, eyelid closure, mouth closure, tongue movement and palate elevation $(0=$ normal, $1=$ reduced, 2 = weak, 3 = absent $)$ with a lowest possible score of 14 . Reflexes were summed by counting the number of muscles scoring 3 or 4 on the NINDS reflex scale for the biceps, triceps, brachioradialis, finger flexor, quadriceps and gastrocnemius muscles on both sides and masseter muscle (maximum score 13). Spasticity was described as none (0), noticeable (1), pronounced (2), barely to overcome (3) and not to overcome (4) with a worst score of 32 (arm, hand, upper thigh, lower thigh both sides). A positive Babinski sign was counted separately for each side (worst score of 2). The clinical characteristics of the patients are summarized in Table 1.

\section{Image acquisition}

Images were acquired on a neuro-optimized 1.5-T GE Signa Horizon LX (General Electric Company, Milwaukee, WI, USA) using a 3-dimensional T1-weighted spoiled gradient recalled echo (SPGR) sequence generating 124 contiguous sagittal slices (RT $24 \mathrm{~ms}$; TE $8 \mathrm{~ms}$; flip angle $30^{\circ}, 2$ averages, acquisition time $13^{\prime} 10^{\prime}$, in plane resolution $0.97 \times 0.97 \times 1.5 \mathrm{~mm}^{3}$ ). During scanning, all participants 
Table 1 Clinical data

\begin{tabular}{|c|c|c|c|c|c|c|c|c|c|c|c|c|c|c|}
\hline Pat. & Age & Sex & Dur. & $\begin{array}{l}\text { RUE } \\
\text { (75) }\end{array}$ & $\begin{array}{l}\text { LUE } \\
\text { (75) }\end{array}$ & $\begin{array}{l}\text { RLE } \\
\text { (45) }\end{array}$ & $\begin{array}{l}\text { LLE } \\
(45)\end{array}$ & $\begin{array}{l}\text { Bulbar } \\
(0-14)\end{array}$ & $\begin{array}{c}\text { Babinski } \\
(0-2)\end{array}$ & $\begin{array}{c}\text { Spasticity } \\
(0-32)\end{array}$ & $\begin{array}{c}\text { Reflexes } \\
(0-13)\end{array}$ & ALSFRS-R & ALSFRS-R PR/M6 & ALSFRS-R PR/M(t) \\
\hline 1 & 59 & $\mathrm{~m}$ & 30 & 68 & 69 & 41 & 41 & 2 & 0 & 0 & 0 & 43 & 0.5 & 0.2 \\
\hline 2 & 59 & $\mathrm{~m}$ & 13 & 75 & 64 & 31 & 27 & 0 & 0 & 0 & 0 & 37 & 1.8 & 0.8 \\
\hline 3 & 31 & $\mathrm{~m}$ & 17 & 64 & 62 & 45 & 45 & 0 & 2 & 0 & 12 & 42 & 1.2 & 0.4 \\
\hline 4 & 68 & $\mathrm{~m}$ & 22 & 75 & 73 & 45 & 42 & 2 & 2 & 4 & 4 & 40 & 0.7 & 0.4 \\
\hline 5 & 65 & $\mathrm{~m}$ & 25 & 72 & 75 & 26 & 16 & 0 & 1 & 0 & 6 & 37 & 0.7 & 0.4 \\
\hline 6 & 53 & $\mathrm{~m}$ & 38 & 74 & 75 & 26 & 23 & 0 & 0 & 0 & 0 & 43 & 0.2 & 0.1 \\
\hline 7 & 34 & w & 18 & 70 & 71 & 45 & 45 & 4 & 2 & 0 & 12 & 39 & 1.0 & 0.5 \\
\hline 8 & 62 & $\mathrm{~m}$ & 18 & 63 & 73 & 45 & 45 & 4 & 0 & 0 & 12 & 40 & 0.7 & 0.4 \\
\hline 9 & 49 & $\mathrm{~m}$ & 19 & 54 & 55 & 45 & 45 & 0 & 2 & 0 & 0 & 41 & 0.3 & 0.4 \\
\hline 10 & 60 & $\mathrm{~m}$ & 30 & 58 & 57 & 45 & 45 & 2 & 2 & 0 & 5 & 33 & 1.5 & 0.5 \\
\hline 11 & 77 & $\mathrm{~m}$ & 34 & 70 & 71 & 22 & 37 & 0 & 2 & 2 & 12 & 33 & 1.7 & 0.4 \\
\hline 12 & 63 & w & 12 & 75 & 75 & 45 & 45 & 3 & 0 & 0 & 11 & 45 & 0.2 & 0.3 \\
\hline 13 & 66 & $\mathrm{~m}$ & 23 & 61 & 71 & 44 & 44 & 0 & 1 & 0 & 0 & 48 & 0.0 & 0.0 \\
\hline 14 & 72 & $\mathrm{~m}$ & 25 & 69 & 71 & 40 & 41 & 3 & 2 & 4 & 6 & 48 & 1.0 & 0.3 \\
\hline
\end{tabular}

Pat. = patient; Dur. = duration in months; $m=$ man, $w=$ woman; RUE, LUE, RLE, LLE: right upper, left upper, right lower, left lower extremity; ALSFRS-R: ALS functional rating scale- revised, $\mathrm{PR} / \mathrm{M} 6$ progression rate for the 6 months prior to MR-scanning, $\mathrm{PR} / \mathrm{M}(\mathrm{t})$ progression rate per month for the entire duration of the disease.

were comfortably placed and their heads were fixated within the headcoil with special cushions. All subjects received additional T2-weighted images to exclude any ischemic or inflammatory lesions. These were normal in all participants.

\section{Preprocessing and advanced segmentation analysis}

The entire analysis was performed using Brainvoyager QX 1.8-1.10 (Brain Innovation, Maastricht, The Netherlands). Data was resampled to achieve $1 \times 1 \times 1 \mathrm{~mm}$ resolution, aligned to the AC-PC line and transformed into Talairach standard space. Prior to cortical thickness analysis, data had to be resampled once more to $0.5 \mathrm{~mm}$ iso-voxel using sinc interpolation. The brain was then segmented from surrounding head tissue using an automatic "brain peeling" tool [36] and subcortical structures and the cerebellum were removed using a mask. Tissue contrast and homogeneity were enhanced by converting the grey-scale from 8 bit to 16 bit resolution and by using a sigma filter. The border between white and grey matter was segmented automatically using an adaptive region growing step using locally computed histograms and gradient information. Subsequently, the border between grey matter and cerebrospinal fluid border was segmented using a dilation process beginning at the white matter-grey matter boundary and moving towards the CSF boundary. This process was controlled by computed gradient fields and histogram analysis of grey matter-CSF threshold values. The aforementioned segmentation steps proved to be unreliable in several regions of the brain, leading, for example, to bridges across sulci. Thus, extensive interactive correction was carried out by author JT who was blinded with regard to whether or not a given brain belonged to a patient or a control participant.

\section{Cortical alignment}

To further improve the spatial correspondence between participants' brains, reconstructed cortices were aligned using curvature information reflecting the gyral/sulcal folding pattern. This approach has been shown to substantially reduce anatomical variability [37]. The reconstructed folded cortical representations of each participant were morphed into a spherical representation (separately for each hemisphere) providing a parameterizable surface suitable for across-subject non-rigid alignment. Each vertex on the sphere corresponded to a vertex of the folded cortex. The curvature information was preserved as a curvature map on the spherical representation and was smoothed along the surface to provide spatially extended gradient information for intercortex alignment. The mean squared differences between the curvature of a source and a target sphere were minimized during the iterative alignment process $[36,38]$.

Cortical thickness maps for each subject were calculated and measurements were performed using the Laplace method [23] as implemented in Brain Voyager Qx. For the whole-cortex analysis the correction for multiple comparisons was performed using cluster-size thresholding. The procedure started with setting a threshold (manually) for the map relative to the contrast of interest with a $\mathrm{p}<0.05$ (uncorrected). Next, the intrinsic smoothness of the resulting map was evaluated automatically [36,39]. The final result was a minimum cluster size threshold 
$\left(1 \mathrm{~mm}^{2}\right)$ for the current map to achieve a corrected p value of $<0.045$.

\section{Region-of-interest analysis of cortical thickness maps}

While group differences in cortical thickness measurements can be mapped at the vertex level, the statistical power of this approach is limited because of the multiple comparisons problem. We therefore also explored cortical thickness in certain regions (or more precisely cortical patches) of interest. We used previous functional data and structural data to guide definition of patches of interest which were placed in the left and right pre- and postcentral gyri as well as in the left and right superior frontal gyrus and the inferior frontal sulcus. The average cortical thickness was determined for each region of interest (ROI) and participant and results were subjected to statistical analysis using between group t-tests or Pearson correlation.

\section{Results}

\section{Whole cortex analysis}

Results at the vertex level are shown in Figure 1 with blue colors indicating regions with significantly reduced cortical thickness and red regions indicating regions in which cortical thickness was increased with regard to the normal participants.

Table 2 illustrates the cortical regions that showed significantly reduced cortical thickness in ALS. These included regions in the pre- and postcentral gyrus bilaterally, the SPL bilaterally, the insula bilaterally, the cingulate gyrus and sulcus bilaterally and the angular/supramarginal gyri and regions in the temporal and occipitotemporal cortex. Thus, cortical thinning extended well beyond the sensorimotor cortex.

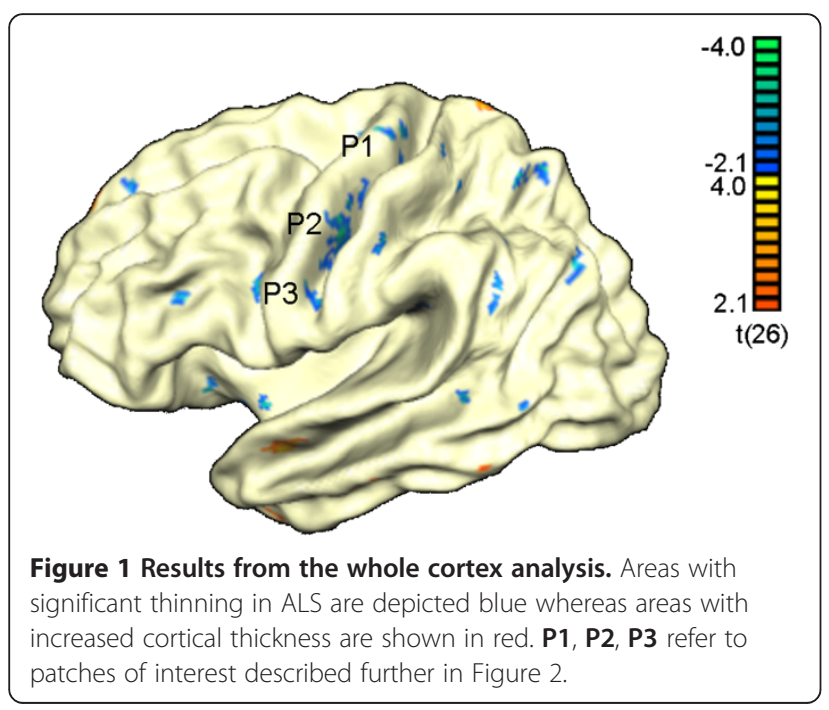

There were few regions showing increased thickness in ALS (see Table 3) including parts of temporal cortex and cingulate cortex.

\section{Region of interest analysis}

Both, left and right precentral cortex showed a highly significant reduction of the cortical thickness in ALS (left: $\mathrm{t}(26)=3.76, \mathrm{p}<0.001$; right: $\mathrm{t}(26)=3.81, \mathrm{p}<0.001$ ). The left and right postcentral gyrus again showed a highly significant reduction of the cortical thickness in ALS (left: $\mathrm{t}(26)=3.24, \mathrm{p}<0.005$; right: $\mathrm{t}(26)=3.51$, $\mathrm{p}<0.003)$. The superior frontal gyrus showed significant thinning in ALS on the left $(\mathrm{t}(26)=3.2, \mathrm{p}<0.005)$ and right $(\mathrm{t}(26)=3.8, \mathrm{p}<0.001)$. The inferior frontal sulcus also showed significant thinning on the left $(t(26)=3.29$, $\mathrm{p}<0.003)$ and right $(\mathrm{t}(26)=2.36, \mathrm{p}<0.03)$.

To further illustrate the reduction of cortical thickness in the pre- and postcentral gyri and the precentral sulci, we computed cortical thickness for a number of patches (see Figure 2 for location and Figure 3 for numerical results). Interestingly, LH3 which was located in the area of the bulbar representations numerically showed a more pronounced but non-significant reduction in patients with bulbar involvement (1.7 mm compared to $2.1 \mathrm{~mm}$ in nonbulbar patients).

We repeated these ROI analyses with age, sex, and disease duration as covariate using the procedure UNIANOVA of SPSS instead of a $t$-test. This did not change the pattern of results.

\section{Correlations with clinical data}

Cortical thickness of the ROIs showed a correlation with ALSFRS- $\mathrm{R}$ in the right superior frontal gyrus $(\mathrm{r}=-0.534$, $\mathrm{P}<0.05$, Figure 4) and the right inferior frontal gyrus $(\mathrm{r}=-0.535 ; \mathrm{p}<0.05)$. Cortical thickness of the right inferior frontal sulcus correlated with progression score $(\mathrm{r}=0.591 ; \mathrm{p}<0.03)$.

We did not find any systematic relationship between cortical thickness and the scores assessing spasticity and reflexes.

Finally, we also did not observe a correlation between age and cortical thickness in any region.

\section{Discussion}

Expectedly, the current study revealed cortical thinning of the primary motor cortex bilaterally in ALS. Importantly, atrophy was also found for the postcentral gyrus bilaterally demonstrating that ALS extends beyond the motor system. These results resonate well with previous findings using voxel-based morphometry [11-13,40-42]. The current analysis of cortical thickness also pointed to an involvement of additional extramotor areas including, for example, the cingulate cortex, as well as regions in the temporal and occipital cortex. That the CTA findings of 
Table 2 Regions of reduced cortical thickness in ALS compared to control participants

\begin{tabular}{|c|c|c|c|c|c|c|}
\hline Location & \# vertices & Central vertex & $B V X$ & BV Y & BV Z & $\mathrm{BA}$ \\
\hline LH precentral G & 51 & 30255 & 156.2 & 70.2 & 155.4 & 4 \\
\hline LH precentral G & 132 & 7509 & 142.8 & 91.4 & 169.1 & 4 \\
\hline LH precentral G & 24 & 7961 & 137.1 & 104.4 & 177.4 & 6 \\
\hline LH precentral S & 23 & 30468 & 124.1 & 104.7 & 167.6 & 6 \\
\hline LH postcentral G & 43 & 7932 & 141.8 & 98.5 & 176.7 & 1 \\
\hline LH postcentral G & 17 & 27991 & 165.4 & 74.4 & 159.1 & 7 \\
\hline LH postcentral G & 12 & 27754 & 169.4 & 82.3 & 159.5 & 7 \\
\hline LH postcentral G & 21 & 8232 & 151.0 & 92.6 & 176.2 & 40 \\
\hline LH SPL & 39 & 28075 & 186.2 & 80.2 & 152.2 & 7 \\
\hline LH IPL & 17 & 19218 & 196.2 & 99.7 & 162.6 & 39 \\
\hline LH angular G & 17 & 34659 & 178.3 & 92.8 & 169.3 & 39 \\
\hline LH angular G & 27 & 33840 & 176.7 & 102.4 & 176.5 & 39 \\
\hline LH insula & 12 & 12243 & 110.9 & 131.2 & 153.9 & na \\
\hline LH insula & 12 & 779 & 119.3 & 134.5 & 158.6 & na \\
\hline LH insula & 20 & 12358 & 124.7 & 133.1 & 162.5 & na \\
\hline LH supramarginal G & 16 & 33153 & 160.9 & 106.5 & 172.6 & 40 \\
\hline LH uncus & 13 & 38776 & 144.5 & 139.2 & 145.6 & na \\
\hline LH cingulate $S$ & 11 & 37338 & 98.8 & 128.6 & 133.4 & 11 \\
\hline LH cingulate $S$ & 14 & 40060 & 129.0 & 88.1 & 137.6 & 24 \\
\hline LH cingulate G & 13 & 37611 & 96.7 & 116.0 & 134.5 & 24 \\
\hline LH cingulate G & 17 & 25234 & 168.8 & 97.1 & 134.1 & 23 \\
\hline LH calcarine $\mathrm{S}$ & 12 & 22417 & 186.8 & 127.0 & 151.0 & 17 \\
\hline RH precentral G & 23 & 6950 & 166.0 & 64.9 & 122.5 & 4 \\
\hline RH precentral S & 17 & 29332 & 136.2 & 80.8 & 100.8 & 4 \\
\hline RH postcentral G & 19 & 1638 & 160.1 & 85.3 & 86.0 & 7 \\
\hline RH postcentral G & 56 & 24668 & 152.6 & 94.7 & 78.2 & 40 \\
\hline RH inf. frontal S & 15 & 37703 & 94.7 & 115.1 & 89.2 & 44 \\
\hline RH SPL & 15 & 1788 & 183.9 & 76.5 & 103.2 & 7 \\
\hline RH lat. occipitotemp. G & 16 & 21928 & 198.1 & 140.5 & 88.1 & 37 \\
\hline RH inf. temporal G & 15 & 22236 & 196.8 & 132.1 & 83.1 & 20 \\
\hline $\mathrm{RH}$ sup. temporal $\mathrm{S}$ & 14 & 21134 & 149.6 & 135.8 & 79.0 & 21 \\
\hline RH insula & 31 & 5792 & 155.4 & 109.9 & 94.8 & na \\
\hline RH lateral S & 19 & 6021 & 160.5 & 120.7 & 80.3 & 42 \\
\hline RH lateral S & 11 & 25291 & 170.9 & 105.4 & 80.5 & 40 \\
\hline RH lateral S & 14 & 23138 & 164.4 & 113.9 & 91.1 & 42 \\
\hline RH lateral S & 67 & 39268 & 138.9 & 132.5 & 91.0 & na \\
\hline RH sup. frontal G & 48 & 2234 & 95.3 & 99.8 & 120.4 & $8 / 9 / 10$ \\
\hline RH cingulate $\mathrm{G}$ & 11 & 11154 & 175.3 & 114.5 & 122.5 & 30 \\
\hline $\mathrm{RH}$ precuneus & 21 & 17430 & 182.7 & 120.3 & 120.9 & 31 \\
\hline $\mathrm{RH}$ calcarine $\mathrm{S}$ & 17 & 17781 & 185.8 & 125.9 & 104.8 & 17 \\
\hline RH med. occipitotemp. G & 15 & 1082 & 197.2 & 131.8 & 118.9 & 19 \\
\hline RH med. occipitotemp. G & 24 & 17728 & 178.6 & 135.0 & 105.4 & 19 \\
\hline RH lat. occipitotemp. G & 45 & 16368 & 168.8 & 145.6 & 95.5 & $20 / 37$ \\
\hline
\end{tabular}

LH, RH: left, right hemisphere; size of the region is given as number of vertices, regions with 10 or less vertices are left out; the number of the central vertex and its $x, y, z$ coordinates as provided by Brain Voyager are also given; BA: Brodmann area; na: not available; G: gyrus; S: sulcus. 
Table 3 Regions of increased cortical thickness in ALS compared to control participants

\begin{tabular}{|c|c|c|c|c|c|c|}
\hline Location & \# vertices & Central vertex & BV $\mathrm{X}$ & BV Y & BV Z & BA \\
\hline LH sup. temporal G & 34 & 2693 & 129.7 & 137.9 & 177.8 & 38 \\
\hline LH med. temporal G & 14 & 16528 & 147.6 & 143.4 & 186.0 & 20 \\
\hline LH inf. temporal G & 22 & 14419 & 126.9 & 162.3 & 164.5 & 38 \\
\hline LH sup. frontal G & 11 & 37020 & 81.9 & 89.2 & 135.0 & 9 \\
\hline LH SPL & 19 & 27475 & 176.0 & 66.1 & 139.2 & 5 \\
\hline LH cingulate G & 14 & 39937 & 118.3 & 98.4 & 133.0 & 24 \\
\hline LH cingulate G & 23 & 25129 & 158.7 & 102.3 & 132.9 & 23 \\
\hline LH precuneus & 17 & 21857 & 177.7 & 123.3 & 137.1 & 17 \\
\hline LH cingulate G & 31 & 22280 & 167.9 & 129.8 & 140.3 & 30 \\
\hline LH hippocampus & 39 & 13505 & 130.4 & 136.9 & 150.0 & na \\
\hline RH insula & 12 & 38050 & 119.0 & 132.1 & 97.3 & na \\
\hline RH orbital G & 20 & 34931 & 106.5 & 37.6 & 101.2 & 11 \\
\hline RH cingulate G & 79 & 17828 & 170.9 & 128.0 & 116.6 & 30 \\
\hline
\end{tabular}

$\mathrm{LH}, \mathrm{RH}$ : left, right hemisphere; size of the region is given as number of vertices, regions with 10 or less vertices are left out; the number of the central vertex and its $x, y, z$ coordinates as provided by Brain Voyager are also given; BA: Brodmann area; na: not available; G: gyrus; S: sulcus.

the current study are not spurious is suggested by other recent studies [28-30] as well as by reports of functional changes implicating the same areas. For example, marked alterations of early visual event-related potential components [43] show that visual cortical areas are indeed altered in the ALS disease process. Also, impaired initiation and inhibition of movements implies the supplementary motor area and right prefrontal cortex $[8,44,45]$.

These findings could guide further neuropathological investigations in ALS in order to pinpoint the underlying histological changes. A marked loss of large pyramidal cells and Betz cells in conjunction with astrocytic gliosis has been described in motor cortex in ALS $[25,46]$. Other neuropathological studies have shown loss of parvalbuminpositive interneurons in ALS irrespective of the degree of loss of Betz-cells in the motor cortex [47]. Loss of GABAergic interneurons in the primary motor cortex and beyond (dorsolateral prefrontal cortex and anterior cingulate cortex) has also been documented [48]. According to the present data extramotor involvement in ALS is
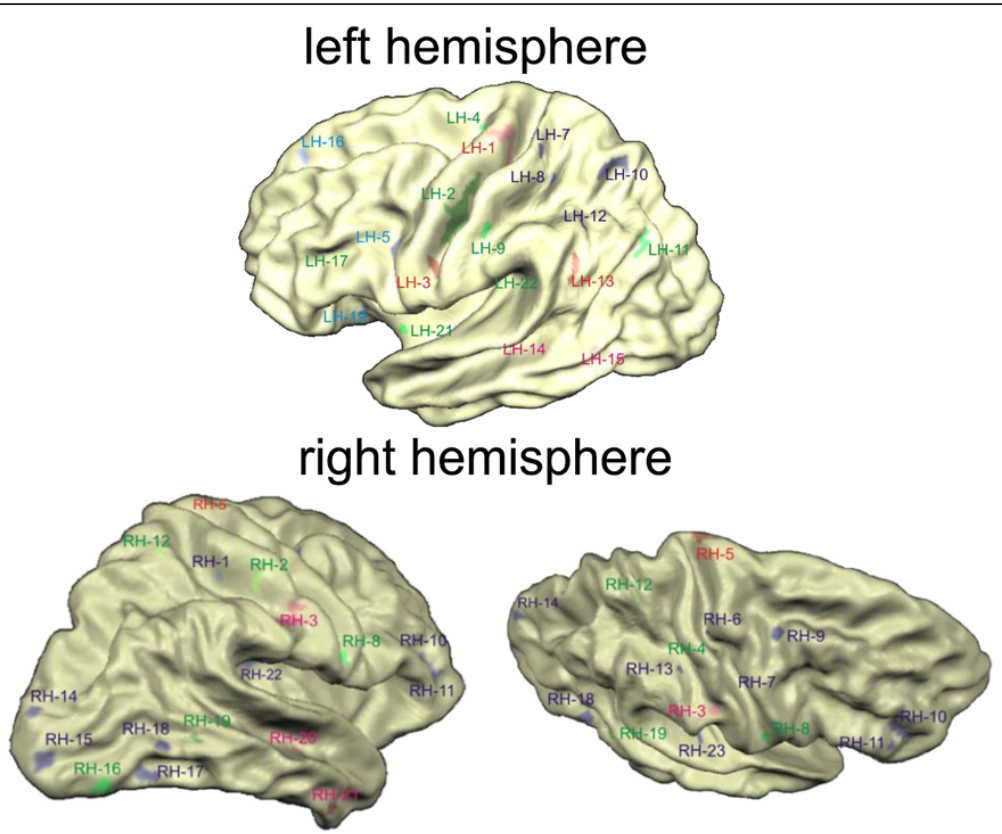

Figure 2 Views of the left (upper part) and right (lower part) hemispheres with labels for the patches that showed significantly reduced cortical thickness in ALS patients. See Figure 3 for results for selected patches. 




widespread also involving brain areas in the frontal, parietal, temporal and occipital lobe. It remains to be seen whether neuropathological changes are equivalent in all of these regions. Also, it is important to note that the patients involved in the current study were affected only mildly or moderately. Application of cortical thickness analysis in more advanced disease is therefore warranted.

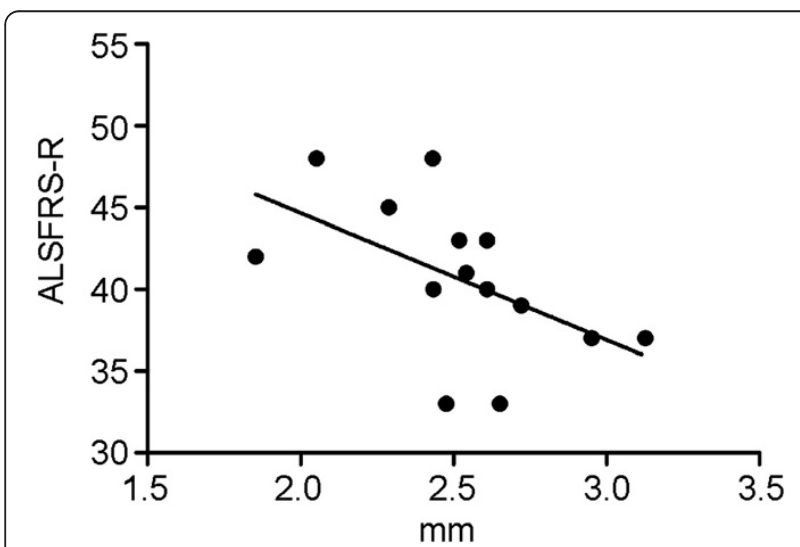

Figure 4 Relation of clinical severity and cortical thickness of the right frontal gyrus. There was a negative correlation between ALSFRS-R score and cortical thickness.
A previous study using MRI-based cortical thickness analysis in ALS used a region-of-interest approach rather than a whole cortex analysis [27]. In contrast to the present results, this study found a significant thinning of the precentral but not postcentral gyrus in ALS. The thickness of the precentral gyrus was determined to be $2.15 \mathrm{~mm}$ in normal controls which compares to about $2.8 \mathrm{~mm}$ found in neuropathological studies [26]. The current data more closely resemble numbers usually found in post-mortem studies. Another recent study investigated primary lateral sclerosis and focussed on cortical thickness of the primary motor cortex in the area of the hand-knob [31]. Patients showed thinning of the motor cortex but not of the primary sensory cortex. The present investigation as well as other recent studies [28-30] significantly extends these previous findings by using a whole cortex approach supplemented by regions of interest analysis.

Interestingly, cortical thickness in the right superior frontal gyrus and in the right inferior frontal gyrus showed a negative correlation with the ALSFRS-R score. This is counter-intuitive, as lower ALSFRS-R scores indicate worse clinical status and, thus, one would expect lower scores to be associated with cortical thinning. It has to be kept in mind, however, that the ALSFRS-R score assesses global functionality of the patient with a strong focus on motor functions and that the brain areas which showed correlations in the present study were not primary motor. In fact, we did not observe any correlations of the ALSFRS-R score to cortical thickness in the precentral gyrus. A recent study investigated cortical thickness and used scores for upper motor neuron and lower motor neuron involvement [31]. In this study, a regionally specific thinning (e.g., bulbar part of the motor cortex for bulbar symptoms) was described in correlation with upper motor neuron signs.

A few brain regions showed an increase of cortical thickness in ALS, some of them even adjacent to regions showing thinning. None of the VBM studies published on ALS reported increases of grey matter density and it is unclear whether such changes were assessed. While the whole cortex statistics used in the present study were properly safeguarded against type 1 error by correction for multiple comparisons using cluster-size thresholding, it is not impossible that one or the other brain area with increased cortical thickness represents a "false positive". However, we do not believe that this can explain the observed pattern. Interestingly, the areas with increased thickness are located mainly in temporal and medial frontal cortex (parts of the cingulate cortex). One possibility is that the observed increases are related to compensatory mechanisms. For example, we have observed an increase of cortical thickness in brain areas related to visuospatial processing and memory in subjects that did not learn to read (unpublished), which we 
took as indicating a greater reliance of illiterates on visuospatial and memory processes. An interplay between frontal and temporal structures is often seen in functional imaging studies of tasks involving executive and working memory functions [49-52] and changes in anatomical connectivity between frontal and temporal structures have been documented in ALS [15]. Moreover, a pattern of hypo- and hyperactivation in a motor task has been observed recently in relation to cortical atrophy in ALS [53]. If structural loss (cortical thinning) in one part of this system is related to structural gain (cortical thickening) in the connected areas remains to be tested in further studies.

\section{Cortical thickness vs. voxel-based morphometry}

This data set has been analyzed previously using VBM [13] which had revealed widespread differences between ALS and normal controls in areas including the right (but not left) precentral gyrus, the postcentral gyrus bilaterally, the inferior parietal lobule bilaterally, and the medial and superior frontal gyri bilaterally. Both, VBM and CTA, showed a correlation between disease severity as reflected by ALSFRS-R and the integrity of the right frontal cortex. CTA appeared more sensitive than VBM in the detection of involvement of extramotor areas. Also, in contrast to the VBM-study the current analysis revealed the left primary motor cortex and supplementary motor cortex. This suggests that cortical thickness analysis is more sensitive to subtle changes of cortical anatomy than VBM. Bermudez et al. [54] similarly reported on differences between VBM and CTA in a study of anatomical changes in professional musicians and underscored the utility of CTA.

One further advantage of CTA is the fact that it measures a meaningful parameter, i.e. cortical thickness, that readily compares to measurements obtained in neuropathological specimens. The fact that the current measurements (about $2.9 \mathrm{~mm}$ for the motor cortex in normal participants) were almost identical to values obtained using neuropathological methodology [26] is reassuring. By contrast, VBM determines grey matter density and it is unclear how this translates into actual anatomical changes [54]. On the other hand, determination of cortical thickness is considerably more time-consuming and requires more user interaction than VBM which can be highly automatized.

\section{Limitations and prospects}

Several limitations of the current study should be mentioned. First, the number of participants per group was relatively small and thus the whole cortex analysis obviously is problematic. A larger data base of normal participants and patients would be desirable. This could be used to test the cortical thickness of individual patients in selected regions of interest such as the pre- and postcentral gyri against the distribution of the group, for example using the test statistic proposed by Crawford and Garthwaite [55]. Such an approach might prove clinically useful in cases in which involvement of the upper motor neuron is not yet apparent. To capture the dynamics of the disease, longitudinal studies are desirable. Recently, tools have been developed to assess changes of cortical thickness over time [56].

\section{Conclusions}

The present study adds to previous investigations in showing that the cortical involvement in ALS extends considerably beyond the motor system. The task for the future is to establish a relation between this involvement and motor, cognitive, and emotional deficits in ALS. Initial steps in this direction have already been taken in a recent publication [57].

\section{Abbreviations}

ALS: amyotrophic lateral sclerosis; ALSFRS-R: amyotrophic lateral sclerosis functional rating scale - revised; CTA: cortical thickness analysis; MRC: medical research council; MRI: magnetic resonance imaging; VBM: voxel-based morphometry.

\section{Competing interests}

The authors declare that the research was conducted in the absence of any commercial or financial relationships that could be construed as a potential competing interest.

\section{Authors' contributions}

JT: Performed the analyses, wrote first draft of the manuscript. HJ: participated in the analysis and revised the manuscript critically for important intellectual content. TP: recorded the data and revised the manuscript critically for important intellectual content. JG: recruited the patients, performed the clinical staging and revised the manuscript critically for important intellectual content. BM: participated in the analyses and revised the manuscript critically for important intellectual content. RD: participated in patient recruitment, study design and revised the manuscript critically for important intellectual content. TFM: conceived the study and wrote the final version of the manuscript. All authors read and approved the final manuscript.

\section{Acknowledgments}

The work was supported by grants from the DFG and the BMBF to TFM.

\section{Author details}

'Department of Neurology, University of Lübeck, Ratzeburger Allee 160, 23538 Lübeck, Germany. ${ }^{2}$ Department of Psychiatry, Medical School Hannover, Hannover, Germany. ${ }^{3}$ Department of Neurology, University of Jena, Jena, Germany. ${ }^{4}$ Department of Neurology and Clinical Neurophysiology, Medical School of Hannover, Hannover, Germany.

Received: 29 December 2012 Accepted: 14 October 2013 Published: 18 October 2013

\section{References}

1. Barson FP, Kinsella GJ, Ong B, Mathers SE: A neuropsychological investigation of dementia in motor neurone disease (MND). J Neurol SCi 2000, 180:107-113.

2. Frank B, Haas J, Heinze HJ, Stark E, Münte TF: Relation of neuropsychological and magnetic resonance findings in amyotrophic lateral sclerosis: Evidence for subgroups. Clin Neurol Neurosurg 1997, 99:79-86.

3. Kew JJM, Goldstein LH, Leigh PN, Abrahams S, Cosgrave N, Passingham RE, et al: The relationship between abnormalities of cognitive function and 
cerebral activation in amyotrophic lateral sclerosis: a neuropsychological and positron emission tomography study. Brain 1993, 116:1399-1423.

4. Massman PJ, Sims J, Cooke N, Haverkamp LJ, Appel V, Appel SH: Prevalence and correlates of neuropsychological deficits in amyotrophic lateral sclerosis. J Neurol Neurosurg Psych 1996, 61:450-455.

5. Abrahams S, Goldstein LH, Simmons A, Brammer M, Williams SCR, Giampietro V, et al: Word retrieval in amyotrophic lateral sclerosis: a functional magnetic resonance imaging study. Brain 2004, 127:1507-1517.

6. Konrad C, Henningsen H, Bremer J, Mock B, Deppe M, Buchinger C, et al: Pattern of cortical reorganization in amyotrophic lateral sclerosis: a functional magnetic resonance imaging study. Exp Brain Res 2002, 143:51-56.

7. Mohammadi B, Kollewe K, Samii A, Krampfl K, Dengler R, Munte TF: Changes of resting state brain networks in amyotrophic lateral sclerosis. Exp Neurol 2009, 217:147-153.

8. Thorns J, Wieringa BM, Mohammadi B, Hammer A, Dengler R, Munte TF: Movement initiation and inhibition are impaired in amyotrophic lateral sclerosis. Exp Neurol 2010, 224:389-394. Neuroimaging in amyotrophic lateral sclerosis.

9. Turner MR, Agosta F, Bede P, Govind V, Lulé D, Verstraete E: Neuroimaging in amyotrophic lateral sclerosis. Biomark Med 2012, 6:319-337.

10. van Blitterswijk M, Dejesus-Hernandez M, Niemantsverdriet E, Murray ME, Heckman MG, Diehl NN, Brown PH, Baker MC, Finch NA, Bauer PO, Serrano G, Beach TG, Josephs KA, Knopman DS, Petersen RC, Boeve BF, Graff-Radford NR, Boylan KB, Petrucelli L, Dickson DW, Rademakers R: Association between repeat sizes and clinical and pathological characteristics in carriers of C9ORF72 repeat expansions (Xpansize-72): a cross-sectional cohort study. Lancet Neurol 2013. 10.1016/S1474-4422(13)70210-2.

11. Kassubek J, Unrath A, Huppertz HJ, Lulé D, Ethofer T, Sperfeld AD, et al: Global brain atrophy and corticospinal tract alterations in ALS, as investigated by voxel-based morphometry of 3-D MRI. Amyotrop Lat Sclerosis Motor Neuron Disord 2005, 6:213-220.

12. Chang JL, Lomen-Hoerth C, Murphy J, Henry RG, Kramer JH, Miller BL, et al: A voxel-based morphometry study of patterns of brain atrophy in ALS and ALS/FTLD. Neurol 2005, 65:75-80.

13. Grosskreutz J, Kaufmann J, Frädrich J, Dengler R, Heinze HJ, Peschel T: Widespread sensorimotor and frontal cortical atrophy in Amyotrophic Lateral Sclerosis. BMC Neurol 2006, 6:17.

14. Ellis CM, Suckling J, Amaro J, Bullmore ET, Simmons A, Williams SCR, et al: Volumetric analysis reveals corticospinal tract degeneration and extramotor involvement in ALS. Neurol 2001, 57:1571-1578.

15. Abrahams $\mathrm{S}$, Goldstein $L H$, Suckling J, Ng V, Simmons A, Chitnis $X$, et al: Frontotemporal white matter changes in amyotrophic lateral sclerosis. J Neurol 2005, 252:321-331.

16. Ashburner J, Friston KJ: Voxel-based morphometry - the methods. Neuroimage 2000, 11:805-821.

17. Ashburner J, Csernansky JG, Davatzikos C, Fox NC, Frisoni GB, Thompson PM: Computer-assisted imaging to assess brain structure in healthy and diseased brains. Lancet Neurol 2003, 2:79-88.

18. Ashburner J, Friston KJ: Why Voxel-based morphometry should be used. Neuroimage 2001, 14:1238-1243.

19. Good CD, Johnsrude IS, Ashburner J, Henson RNA, Friston K, Frackowiak RSJ: A voxel-based morphometric study of ageing in 465 normal adult human brains. Neuroimage 2001, 14:21-36.

20. Bookstein FL: "voxel-based morphometry" should not be used with imperfectly registered images. Neuroimage 2001, 14:1454-1462.

21. Haidar $\mathrm{H}$, Soul JS: Measurement of cortical thickness in 3D brain MRI data: Validation of the Laplacian method. J Neuroimag 2006, 16:146-153.

22. Han X, Jovicich J, Salat D, Van Der Kouwe A, Quinn B, Czanner S, et al: Reliability of MRI-derived measurements of human cerebral cortical thickness: the effects of field strength, scanner upgrade and manufacturer. Neuroimage 2006, 32:180-194.

23. Jones SE, Buchbinder BR, Aharon I: Three-dimensional mapping of cortical thickness using Laplace's equation. Hum Brain Map 2000, 11:12-32.

24. Kabani N, Le Goualher G, MacDonald D, Evans AC: Measurement of cortical thickness using an automated 3-D algorithm: A validation study. Neuroimage 2001, 13:375-380.

25. Brownell B, Oppenheimer DR, Hughes JT: The central nervous system in motor neurone disease. J Neurol Neurosurg Psych 1970, 33:338-357.

26. Toft $\mathrm{MH}$, Gredal $\mathrm{O}$, Pakkenberg B: The size distribution of neurons in the motor cortex in amyotrophic lateral sclerosis. J Anatomy 2005, 207:399-407.
27. Roccataglia L, Bonzano L, Mancardi G, Canepa C, Caponnetto C: Detection of motor cortex thinning and corticospinal tract involvement by quantitative MRI in amyotrophic lateral sclerosis. Amyotrop Lat Scler Motor Neuron Disord 2009, 10:47-52.

28. Verstraete $\mathrm{E}$, Veldink JH, Hendrikse J, Schelhaas HJ, van den Heuvel MP, van den Berg LH: Structural MRI reveals cortical thinning in amyotrophic lateral sclerosis. J Neurol Neurosurg Psych 2012, 83:383-388.

29. Verstraete $\mathrm{E}$, van den Heuvel MP, Veldink JH, Blanken N, Mandl RC, Hulshoff Pol HE, van den Berg LH: Motor network degeneration in amyotrophic lateral sclerosis: a structural and functional connectivity study. PLoS One 2010, 5:e13664.

30. Agosta F, Valsasina P, Riva N, Copetti M, Messina MJ, Prelle A, Comi G, Filippi M: The cortical signature of amyotrophic lateral sclerosis. PLoS One 2012, 7:e42816.

31. Butman JA, Floeter MK: Decreased thickness of primary motor cortex in primary lateral sclerosis. Am J Neuroradiol 2007, 28:87-91.

32. Schuster C, Kasper E, Machts J, Bittner D, Kaufmann J, Benecke R, Teipel S, Vielhaber S, Prudlo J: Focal thinning of the motor cortex mirrors clinical features of amyotrophic lateral sclerosis and their phenotypes: a neuroimaging study. I Neurol 2013. Epub ahead of print.

33. Brooks BR, Miller RG, Swash M, Munsat TL: El Escorial revisited: Revised criteria for the diagnosis of amyotrophic lateral sclerosis. Amyotrop Lat Scler Motor Neuron Disord 2000, 1:293-299.

34. Cedarbaum JM, Stambler N, Malta E, Fuller C, Hilt D, Thurmond B, et al: The ALSFRS-R: a revised ALS functional rating scale that incorporates assessments of respiratory function. J Neurol Sci 1999, 169:13-21.

35. Kollewe K, Mauss U, Krampfl K, Petri S, Dengler R, Mohammadi B: ALSFRS-R score and its ratio: a useful predictor for ALS-progression. J Neurol Sci 2008, 275:69-73.

36. Goebel R, Esposito F, Formisano E: Analysis of Functional Image Analysis Contest (FIAC) data with BrainVoyager QX: From single-subject to cortically aligned group General Linear Model analysis and self-organizing group Independent Component Analysis. Hum Brain Mapp 2006, 27:392-401.

37. Fischl B, Sereno MI, Dale AM: Cortical surface-based analysis: II. Inflation, flattening, and a surface-based coordinate system. Neuroimage 1999, 9:195-207.

38. Formisano E, Esposito F, Di Salle F, Goebel R: Cortex-based independent component analysis of fMRI time series. Magn Reson Imag 2004, 22:1493-1504

39. Forman SD, Cohen JD, Fitzgerald M, Eddy WF, Mintun MA, Noll DC: Improved assessment of significant activation in functional magnetic resonance imaging (fMRI): use of a cluster-size threshold. Magn Reson Med 1995, 33:636-647.

40. Agosta F, Pagani E, Rocca MA, Caputo D, Perini M, Salvi F, et al: Voxel-based morphometry study of brain volumetry and diffusivity in amyotrophic lateral sclerosis patients with mild disability. Hum Brain Mapp 2007, 28:1430-1438

41. Thivard L, Pradat PF, Lehericy S, Lacomblez L, Dormont D, Chiras J, et al: Diffusion tensor imaging and voxel based morphometry study in amyotrophic lateral sclerosis: relationships with motor disability. J Neurol Neurosurg Psych 2007, 78:889-892.

42. Grosskreutz J, Peschel T, Unrath A, Dengler R, Ludolph AC, Kassubek J: Whole brain-based computerized neuroimaging in ALS and other motor neuron disorders. Amyotrop Lat Scler 2008, 9:238-248.

43. Munte TF, Troger MC, Nusser I, Wieringa BM, Johannes S, Matzke M, et al: Alteration of early components of the visual evoked potential in amyotrophic lateral sclerosis. J Neurol 1998, 245:206-210.

44. Riva N, Falini A, Inuggi A, Gonzalez-Rosa JJ, Amadio S, Cerri F, et al: Cortical activation to voluntary movement in amyotrophic lateral sclerosis is related to corticospinal damage: electrophysiological evidence. Clin Neurophysiol 2012, 123:1586-1592.

45. Westphal KP, Heinemann HA, Grozinger B, Kotchoubey BJ, Diekmann V, Becker W, et al: Bereitschaftspotential in amyotrophic lateral sclerosis ALS: lower amplitudes in patients with hyperreflexia spasticity. Acta Neurol Scand 1998, 98:15-21.

46. Hughes JT: Pathology of amyotrophic lateral sclerosis. Adv Neurol 1982, 36:61-74.

47. Nihei K, McKee AC, Kowall NW: Patterns of neuronal degeneration in the motor cortex of amyotrophic lateral sclerosis patients. Acta Neuropathol 1993, 86:55-64. 
48. Maekawa S, Al-Sarraj S, Kibble M, Landau S, Parnavelas J, Cotter D, et al: Cortical selective vulnerability in motor neuron disease: a morphometric study. Brain 2004, 127:1237-1251.

49. Asari T, Konishi S, Jimura K, Chikazoe J, Nakamura N, Miyashita Y: Amygdalar modulation of frontotemporal connectivity during the inkblot test. Psychiatry Res 2010, 182:103-110.

50. Konrad A, Vucurevic G, Musso F, Stoeter P, Dahmen N, Winterer G: ErbB4 genotype predicts left frontotemporal structural connectivity in human brain. Neuropsychopharmacol 2009, 34:641-650.

51. Ragland JD, Gur RC, Valdez J, Turetsky BI, Elliott M, Kohler C, et al: Event-related $\mathrm{fMRI}$ of frontotemporal activity during word encoding and recognition in schizophrenia. Am J Psych 2004, 161:1004-1015.

52. Winterer G, Coppola R, Egan MF, Goldberg TE, Weinberger DR: Functional and effective frontotemporal connectivity and genetic risk for schizophrenia. Biol Psych 2003, 54:1181-1192.

53. Cosottini M, Pesaresi I, Piazza S, Diciotti S, Cecchi P, Fabbri S, Carlesi C, Mascalchi M, Siciliano G: Structural and functional evaluation of cortical motor areas in Amyotrophic Lateral Sclerosis. Exp Neurol 2012, 234:169-180.

54. Bermudez P, Lerch J, Evans AC, Zatorre RJ: Neuroanatomical correlates of musicianship as revealed by cortical thickness and voxel-based morphometry. Cereb Cortex 2009, 19:1583-1596.

55. Crawford JR, Garthwaite PH: Investigation of the single case in neuropsychology: confidence limits on the abnormality of test scores and test score differences. Neuropsychologia 2002, 40:1196-1208.

56. Nakamura K, Fox R, Fisher E: CLADA: Cortical longitudinal atrophy detection algorithm. Neuroimage 2010, 54:278-289.

57. Schuster C, Kasper E, Dyrba M, Machts J, Bittner D, Kaufmann J, Mitchell AJ, Benecke R, Teipel S, Vielhaber S, Prudlo J: Cortical thinning and its relation to cognition in amyotrophic lateral sclerosis. Neurobiol Aging 2014, 35:240-246.

doi:10.1186/1471-2377-13-148

Cite this article as: Thorns et al.: Extent of cortical involvement in amyotrophic lateral sclerosis - an analysis based on cortical thickness. BMC Neurology 2013 13:148.

\section{Submit your next manuscript to BioMed Central and take full advantage of:}

- Convenient online submission

- Thorough peer review

- No space constraints or color figure charges

- Immediate publication on acceptance

- Inclusion in PubMed, CAS, Scopus and Google Scholar

- Research which is freely available for redistribution 